\title{
Distribución de los vectores de Leishmania infantum (Kinetoplastida: Trypanosomatidae) en Colombia
}

\author{
Camila González ${ }^{1}$, Olga L. Cabrera ${ }^{1}$, Leonard E. Munstermann ${ }^{2}$, Cristina Ferro ${ }^{1}$ \\ ${ }^{1}$ Laboratorio de Entomología, Instituto Nacional de Salud, Bogotá, D. C., Colombia. \\ 2 Epidemiology and Public Health Department, Yale University School of Medicine, New Haven, Connecticut, \\ USA.
}

Introducción. Debido a la importancia que tiene la vigilancia entomológica como principal medida de control en el manejo de la leishmaniasis visceral, es necesario contar con información actualizada acerca de la distribución y ecología de los insectos involucrados en la transmisión para optimizar las estrategias de prevención.

Objetivo. Presentar la distribución actualizada geo-referenciada de L. longipalpis y L. evansi, vectores de los parásitos que causan leishmaniasis visceral en Colombia, teniendo en cuenta la asociación de los insectos con su hábitat.

Materiales y métodos. Los registros de distribución se obtuvieron a partir de los ejemplares recolectados en Colombia desde 1967. La información obtenida se organizó en una base de datos a partir de la cual se tomaron las localidades que, posteriormente, fueron sometidas a análisis geográficos por medio de Arc View que se utilizaron para realizar los mapas de distribución.

Resultados. Para L. longipalpis se obtuvieron 40 localidades todas distribuidas a lo largo del valle del río Magdalena: Alto (24), Medio (11) y Bajo (5) Magdalena. L. evansi fue registrado en 19 localidades también ubicadas en el mismo valle: cinco en el Magdalena Medio y 14 el Magdalena Bajo.

Conclusiones. Ambas especies demostraron una consistente asociación con regiones clasificadas principalmente como bosque seco tropical según las zonas de vida de Holdridge lo que confirma el riesgo epidemiológico de leishmaniasis visceral en estas áreas.

Palabras clave: Psychodidae, leishmaniasis visceral, Colombia.

\section{Distribution of Leishmania infantum vector species in Colombia.}

Introduction. Since entomological surveillance is the main control strategy for visceral leishmaniasis, updated information on the distribution and ecology of involved vector species is necessary for planning preventive measures.

Objective. To present the updated and geo-referenced distribution of $L$. longipalpis and $L$. evansi, vectors of visceral leishmaniasis in Colombia, considering their relationship with their habitat.

Materials and methods. Distribution was estimated from records of the sand fly specimens collected since 1967. The information was organized in a database from which the localities were selected and geographically analyzed with Arc view in order to develop the distribution maps.

Results. 40 localities were established for L. longipalpis along the upper (24), middle (11) and lower (5) Magdalena river valley. L. evansi was recorded in 19 localities of the middle (5) and lower (14) Magdalena valley.

Conclusions. Both species showed consistent association with dry tropical forest (sensu Holdridge 1967), confirming the epidemiological risk for visceral leishmaniasis in these areas.

Key words: Psychodidae, leishmaniasis, visceral, Colombia. 
La leishmaniasis visceral es una enfermedad endémica de 61 países que puede ser mortal en ausencia de tratamiento. Su incidencia anual es de cerca de 500.000 casos de los cuales el $90 \%$ se presenta en Bangladesh, India, Nepal y Brasil (1). Las grandes epidemias ocurridas recientemente en África e India y la emergencia de casos en pacientes infectados con VIH han hecho de la leishmaniasis visceral una enfermedad prioritaria para la Organización Mundial de la Salud (2).

Mundialmente, dos especies de parásitos, Leishmania donovani y Leishmania infantum (generalmente considerado como Leishmania chagasi en Latinoamérica $(3,4))$ son los agentes etiológicos responsables de la enfermedad. Un variado número de reservorios (pequeños mamíferos) y diferentes vectores (especies de dípteros de los géneros Phlebotomus y Lutzomyia) son los encargados de mantener los ciclos enzoóticos de la enfermedad en distintas regiones del mundo (4-8). El perro es considerado el principal reservorio doméstico (9).

En el Nuevo Mundo, Lutzomyia longipalpis es el vector principal y se distribuye en áreas tropicales desde el sur de México hasta el norte de Argentina $(10,11)$. En la década de los noventa, otras dos especies fueron incriminadas como vectores de este parásito, L. evansi en Colombia y en Brasil, Lutzomyia cruzi $(12,13)$. L. evansi está presente en Colombia, Venezuela, México, Guatemala, El Salvador, Honduras, Nicaragua y Costa Rica $(10,14,15)$ y L. cruzi en Brasil y Bolivia (13). La presencia de $L$. longipalpis y $L$. evansi puede coincidir en una misma zona (14-18); en el estado de Aragua, Venezuela, la mayor abundancia para L. evansise registra al final de la estación lluviosa, mientras que para $L$. longipalpis el aumento se registra durante la estación seca, lo que sugiere una alternancia estacional (18).

Los estudios de competencia y capacidad vectorial han demostrado que $L$. evansitiene un

\footnotetext{
Correspondencia:

Cristina Ferro, Instituto Nacional de Salud, Avenida Calle

26 N 51-60, Bogotá, D. C. Colombia.

Teléfono: (057-1) 2200923

mferro@ins.gov.co; crisferro@yahoo.com

Recibido: 10/08/05; aceptado: 24/02/06
}

menor potencial de transmisión con respecto a $L$. longipalpis debido a que registra bajas tasas de infección (19-20). Las dos especies han sido asociadas a la transmisión domiciliaria en áreas urbanas y suburbanas $(18,21)$. Además, en Colombia, en años recientes, se encontró $L$. evansi en una zona urbana de la Costa Atlántica (22).

La actualización de la distribución de estos vectores en Colombia permite optimizar las estrategias de prevención definiendo zonas de riesgo y áreas prioritarias de control. Con este fin, en diferentes proyectos de salud pública se están utilizando los sistemas de información geográfica que permiten identificar, clasificar y organizar las variables ambientales involucradas en la distribución y abundancia de las enfermedades (23-26).

El presente trabajo introduce esta herramienta en el estudio de los vectores de leishmaniasis visceral en Colombia y presenta, por primera vez, los mapas digitales de distribución de estas especies y su asociación a las zonas de vida de Holdridge, se incluyen registros previamente documentados y algunos nuevos provenientes de estudios realizados en Colombia.

\section{Materiales y métodos}

Se elaboró una base de datos a partir de registros de ejemplares recolectados en Colombia desde 1967, que fueron confirmados y complementados con datos bibliográficos. Toda esta información se sistematizó por medio del programa Epilnfo 2002 y, a partir de los registros obtenidos, se determinaron las localidades en las que cada una de las especies en estudio había sido registrada. Los registros que determinan la presencia de $L$. evansi en el departamento de Caquetá no se incluyeron porque al revisar la colección de referencia del Instituto Nacional de Salud se encontró que corresponden a Lutzomyia nevesiy no a $L$. evansi como se registró inicialmente (14). Tampoco se incluyeron los de Nariño, Valle del Cauca $(10,15)$ y Meta (Molina JA, Jaramillo M, Villegas C, Guhl F. Actualización de la distribución del género Lutzomyia en Colombia. Biomédica 1997;17:152). Estos últimos registros, posiblemente, se originaron por un error de identificación o una contaminación en las herramientas de trabajo. 
A partir de los datos de distribución obtenidos se hallaron las coordenadas de las localidades y se elaboraron los mapas de distribución de los vectores en Colombia por medio del programa ArcView (versión 3.2). Con el fin de realizar los análisis, se utilizaron las siguientes coberturas: división política de Colombia por municipios (Departamento Administrativo Nacional de Estadística, DANE); zonas de vida de Holdridge (Instituto de Hidrología, Meteorología y Estudios Ambientales, IDEAM) y mapa de ecosistemas generales de Colombia (Alexander von Humboldt).

\section{Resultados}

Se ingresaron 203 registros de las especies vectores del parásito agente causal de la leishmaniasis visceral en Colombia. Para la especie L. longipalpis se obtuvieron 167 registros que confirman su presencia en 40 localidades, de las cuales, $37(92,5 \%)$ se referenciaron geográficamente. En el caso de $L$. evansi, de los 36 registros obtenidos de 19 localidades, 17 $(89,5 \%)$ se referenciaron geográficamente (figura 1$)$.

\section{Distribución geográfica de Lutzomyia longipalpis en Colombia}

L. longipalpis se distribuye en el territorio colombiano a lo largo de la región conocida como valle del río Magdalena, desde el departamento del Huila hasta la región Caribe. Se conoce su presencia en el país desde 1967, a partir de capturas realizadas por Cornelius Marinkelle en el municipio de Honda, departamento del Tolima (27). En 1968 se encontraron ejemplares de esta especie en el departamento de Caldas por Morales y Osorno, en el municipio de Victoria, vereda El Llano. Registros posteriores de Osorno en 1970, indicaron su presencia en los departamentos de Huila, $12 \mathrm{~km}$ antes de Neiva, y en Cundinamarca, municipio de Nilo. En 1975 y 1987 se amplió el conocimiento de su distribución al departamento de Norte de Santander, a los municipios de Gramalote, Durania, Zulia y Arboledas (28). En 1982 y 1983 a los departamentos de Santander, municipio de Girón, vereda Palogordo, y Sucre, municipio de Ovejas, veredas El Ojito y Alemania. En la misma década se registró en La Guajira, en

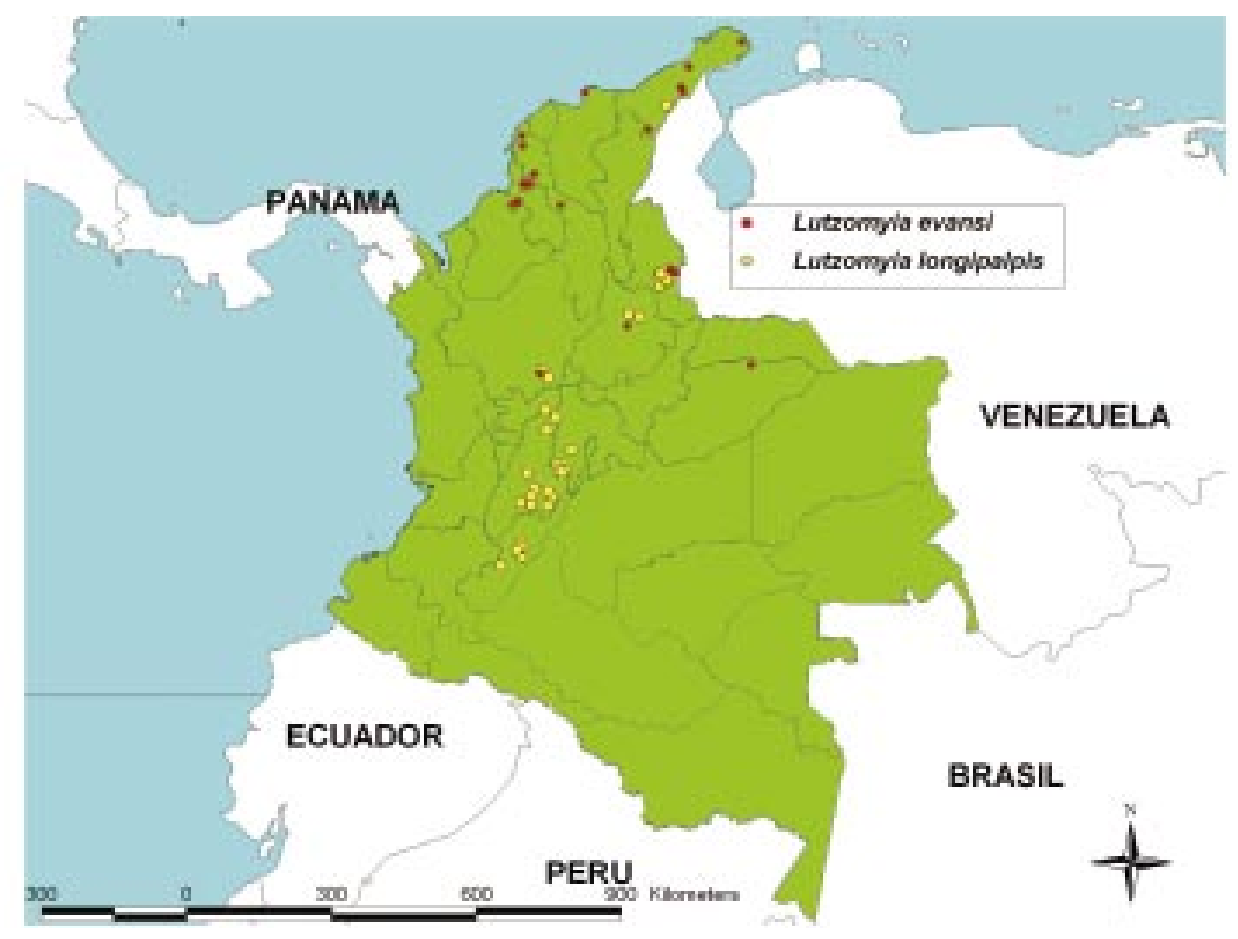

Figura 1. Distribución de Lutzomyia longipalpis y Lutzomyia evansi en Colombia. 
el municipio de Barrancas (29). En 1991, por primera vez, se registra en Antioquia en el municipio de San Luis, veredas La Mulata y El Refugio (30). En el 2002, se capturaron ejemplares en el departamento de Córdoba en el municipio de San Andrés de Sotavento, vereda Villa Nueva (17) (figura 1).

\section{Distribución geográfica de Lutzomyia evansi en Colombia}

De acuerdo con la revisión publicada por Young en 1979 (15), el primer registro de $L$. evansien el país se remonta a especímenes recolectados en 1940 en el departamento de Bolívar en el municipio de Arjona. Posteriormente, en 1944, Gast y Rengifo obtuvieron ejemplares de esta especie procedentes del departamento de Santander en el municipio de San Vicente de Chucurí, vereda Chaparral.

Actualmente, su distribución está confirmada para los departamentos de Antioquia, Casanare, Córdoba, La Guajira, Bolívar, Magdalena, Sucre, Norte de Santander y Santander. En 1969 se registró, por primera vez, en el departamento de La Guajira, a partir de ejemplares recolectados en la vereda Las Marías en el municipio de Urumita. El primer registro que existe para el departamento de Magdalena, está descrito por Young 1979 (15) en 1973 en capturas realizadas en Santa Marta. En el departamento de Norte de Santander se registra en 1983, en el municipio El Zulia, vereda El Guayabo. Los ejemplares recolectados en Córdoba en el municipio de San Andrés de Sotavento y en Sucre en el municipio Colosó, fueron identificados en 1988 y 1997 (10,17, 31) (figura 1). Además, en 2003 se informó de su presencia en el municipio de San Roque, departamento de Antioquia, y en los municipios de Hato Corozal, Paz de Ariporo y Tauramena, en el departamento de Casanare (32); las localidades del departamento de Casanare no se incluyeron en las figuras por no disponer de las coordenadas geográficas.

\section{Distribución de L. longipalpis y L. evansi de acuerdo con las zonas de vida de Holdridge}

De acuerdo con el mapa de zonas de vida de Holdridge (33), L. longipalpis y L. evansi han sido encontrados en Colombia, predominantemente, en regiones con una cobertura vegetal de bosque seco tropical (bs-T), $26(70,3 \%)$ de las 37 localidades para L. longipalpis y $12(75 \%)$ de las 17 para L. evansi. El bs-T se encuentra entre los 0 y $1.000 \mathrm{~m}$ de altitud, tiene una temperatura media superior a los $24^{\circ} \mathrm{C}$ y un rango de precipitación anual entre 1.000 y $2.000 \mathrm{~mm}$ (33); ha sido altamente intervenido debido a la ganadería y el establecimiento de cultivos, quedando un porcentaje muy reducido de su cobertura original (34).

Aunque para $L$. longipalpis la gran mayoría de las localidades están dentro de los límites del bosque seco tropical, algunas se encuentran en otro tipo de cobertura vegetal, a una distancia tan pequeña del bosque seco tropical, que no pudo establecerse con absoluta certeza si efectivamente eran parte de la otra cobertura (figura 2). Por ejemplo, en el departamento de La Guajira, Barrancas fue georreferenciado en la zona de bosque muy seco tropical (bms-T), a una distancia de 2,3 $\mathrm{km}$, aproximadamente, del margen del bs-T. El bms-T tiene un rango de precipitación entre 500 y $1.000 \mathrm{~mm}$ y una temperatura media superior a $24^{\circ} \mathrm{C}$; se encuentra en localidades de La Guajira y Norte de Santander. Este bosque está altamente intervenido debido a que la vegetación nativa ha sido destruida a causa del pastoreo excesivo y a la tala de árboles.

En el departamento de Tolima en los municipios de Dolores y Melgar y en Norte de Santander en el municipio de Gramalote, L. longipalpis fue capturada en áreas correspondientes a bosque húmedo premontano (bh-PM) a una distancia aproximada de $2,5 \mathrm{~km}$ del margen del bs-T. Este tipo de bosque, se encuentra entre los $1.000 \mathrm{y}$ 2.000 metros de altitud, y tiene una temperatura promedio entre 18 y $24^{\circ} \mathrm{C}$ y un rango de precipitación de 1.000 a $2.000 \mathrm{~mm}$ anuales. La vegetación original ha sido explotada para el establecimiento de cultivos y frutales llevando al aumento de la población rural en estas regiones.

En zona de bosque húmedo tropical (bh-T), en el departamento del Tolima, las localidades se ubicaron entre $1,2 \mathrm{~km}$ en Purificación y $11,1 \mathrm{~km}$ en Prado, del límite del bs-T. En Norte de Santander, la localidad de Durania dista $0,7 \mathrm{~km}$ del límite de bs-T. El bh-T se caracteriza por tener 


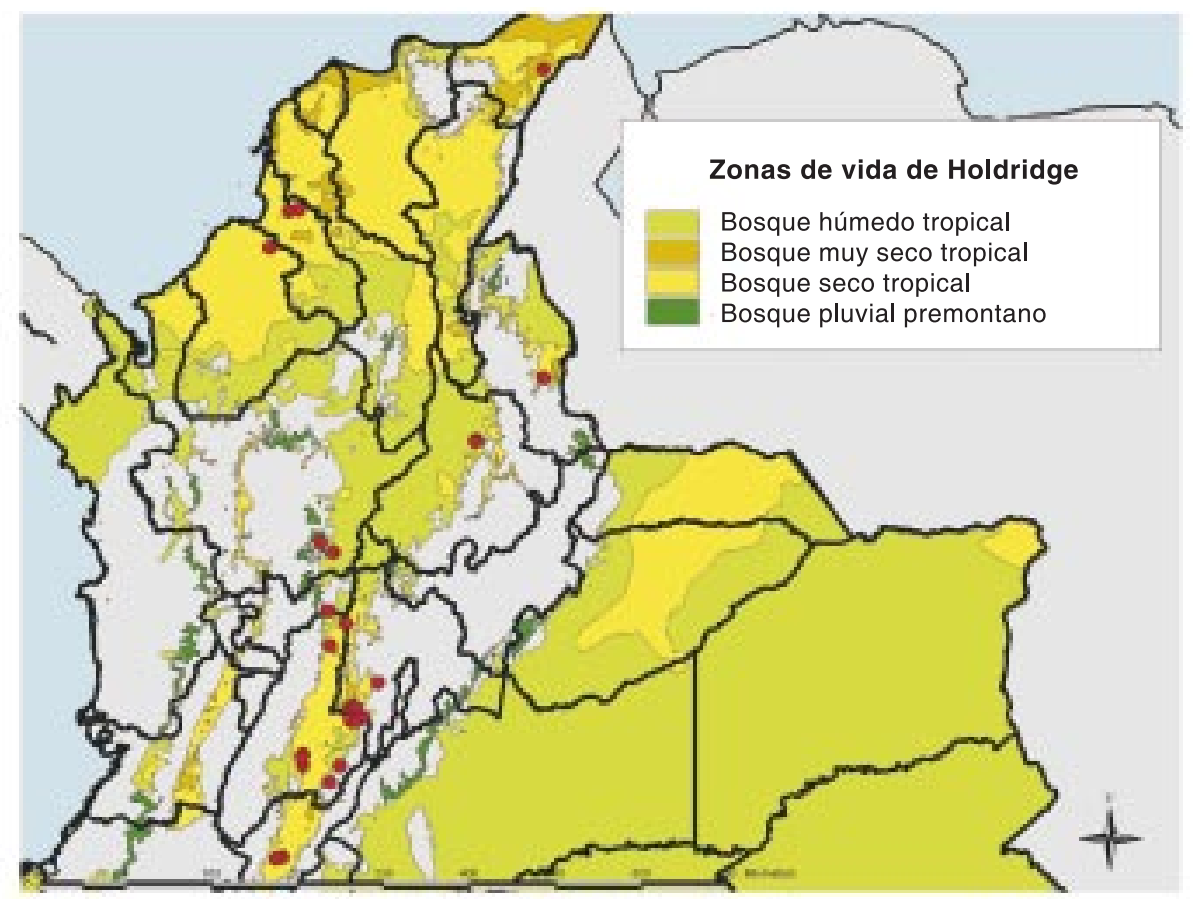

Figura 2. Distribución de Lutzomyia longipalpis de acuerdo con las zonas de vida de Holdridge.

una temperatura superior a $24^{\circ} \mathrm{C}$, promedio anual de lluvias entre 2.000 y $4.000 \mathrm{~mm}$ y se encuentra entre los 0 y 1.000 metros de altitud. Sus suelos, al igual que en el bs-T, son utilizados para ganadería y cultivos. Se conservan regiones con selvas nativas pero que son explotadas para la obtención de maderas. También las localidades de La Mulata en Antioquia y El Llano en Caldas están dentro de la cobertura bh-T.

En cuanto a $L$. evansi, además de las localidades de bs-T, se encontró en zonas que pertenecen a una cobertura de monte espinoso subtropical (meST), caracterizado por tener un rango de precipitación muy bajo $(250$ a $500 \mathrm{~mm})$ y una temperatura media de $24^{\circ} \mathrm{C}$ (35) en el departamento de La Guajira, y en bosque muy seco tropical en localidades de La Guajira y Norte de Santander (figura 3).

Distribución de L. longipalpis y L. evansi de acuerdo con el mapa de ecosistemas generales de Colombia (Etter, 1998)

De acuerdo con el mapa de ecosistemas generales de Colombia (36), la mayoría de las localidades en las que se distribuyen L. longipalpis y $L$. evansi, son áreas altamente intervenidas de las cuales permanece menos del $20 \%$ de la cobertura original. Por ejemplo, en el caso de $L$. longipalpis en Sucre y Cundinamarca y L. evansi en Sucre y Bolívar, los registros corresponden a zonas altamente intervenidas, donde los colonos remplazaron el bosque por la agricultura mixta en las que se encuentran principalmente cultivos y, además, se lleva a cabo el pastoreo (figura 4). Estas zonas intervenidas, en las que las actividades humanas se llevan a cabo en cercanía de las viviendas y hay presencia de los vectores, constituyen áreas de importancia epidemiológica ya que, de introducirse el parásito, podría presentarse transmisión domiciliaria o peridomiciliaria dando origen a nuevos focos de la enfermedad (37). Sólo algunas de las localidades en las que se capturaron insectos se encuentran en ecosistemas relativamente conservados. Este es el caso de L. longipalpis en los departamentos de La Guajira y en Norte de Santander (figura 4) y de L. evansi en los departamentos de La Guajira, Bolívar, Sucre y Norte de Santander (figura 5). 


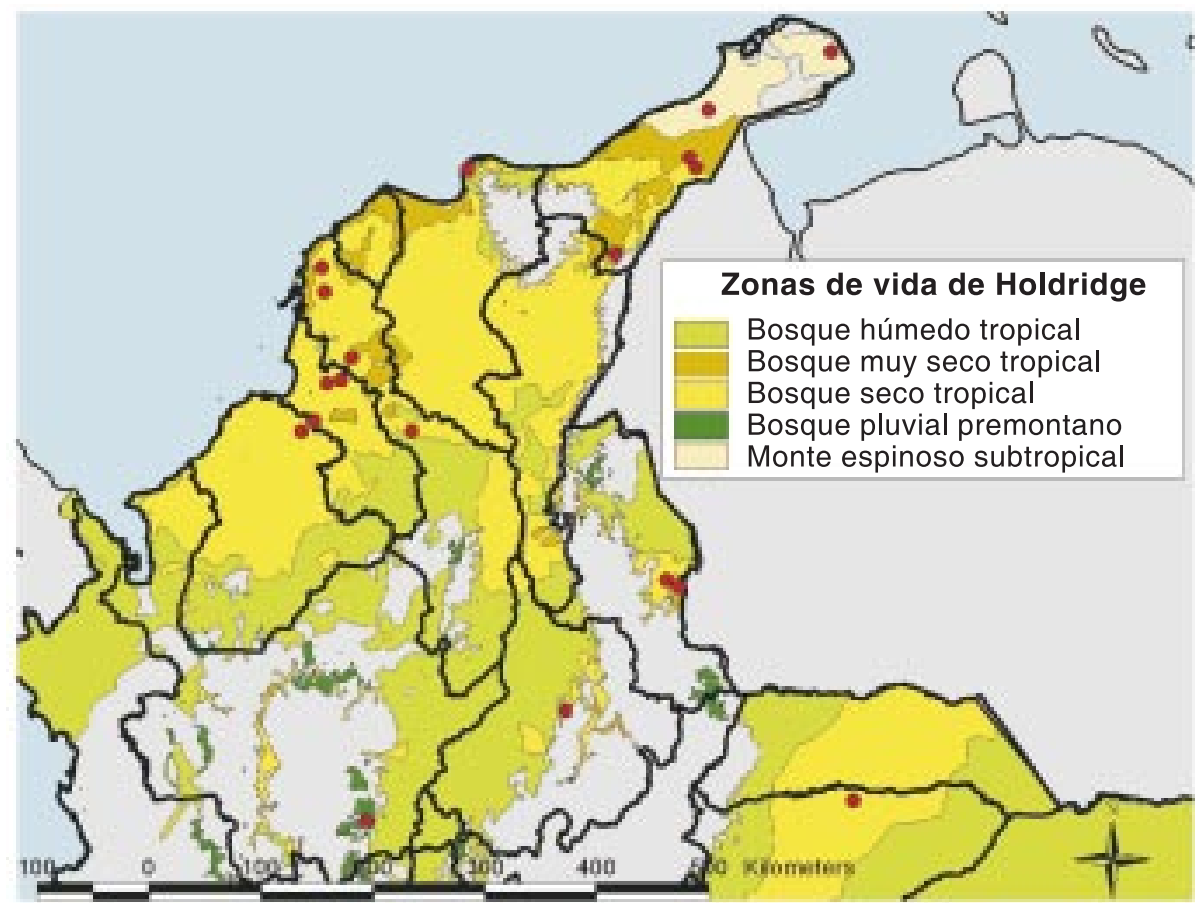

Figura 3. Distribución de Lutzomyia evansi de acuerdo con las zonas de vida de Holdridge.

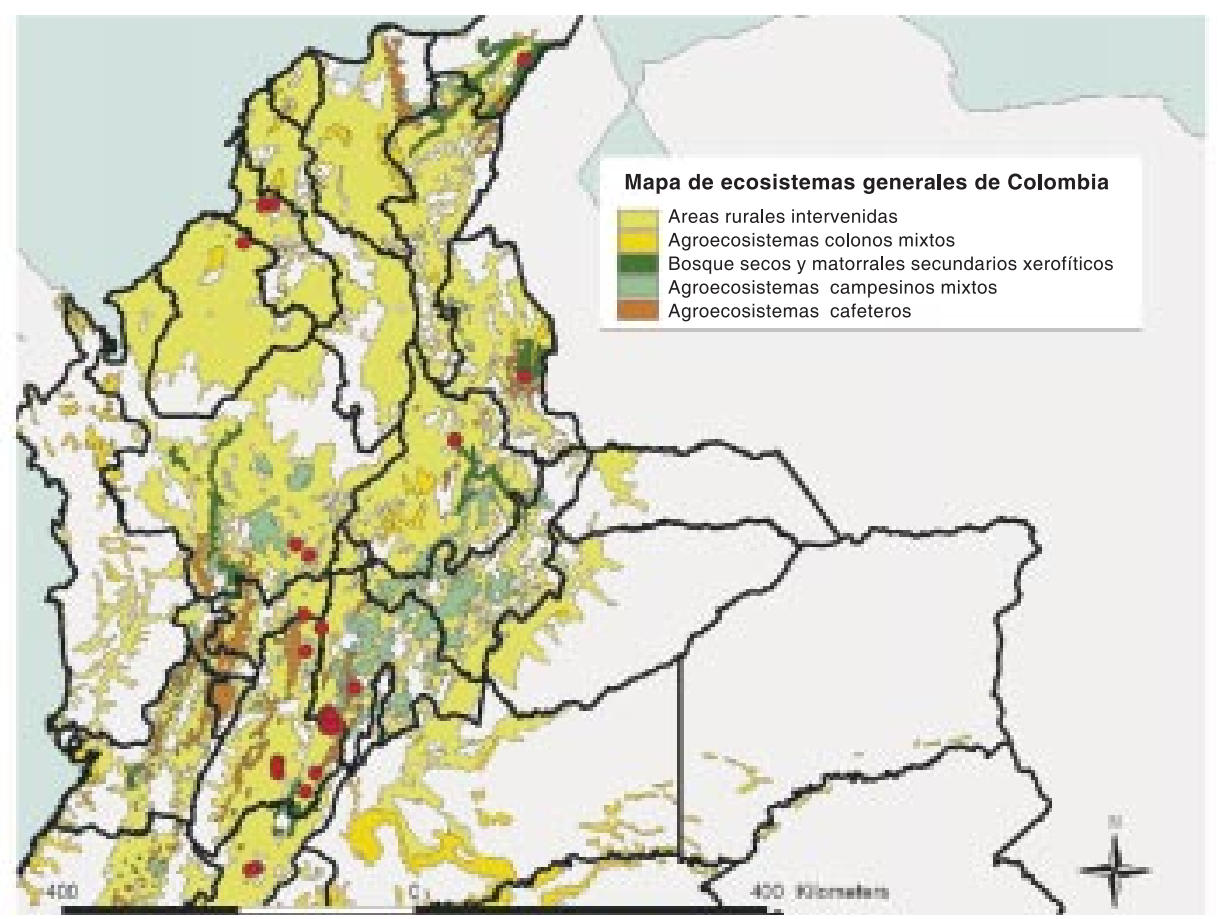

Figura 4. Distribución de Lutzomyia longipalpis de acuerdo con el mapa de ecosistemas generales de Colombia. 


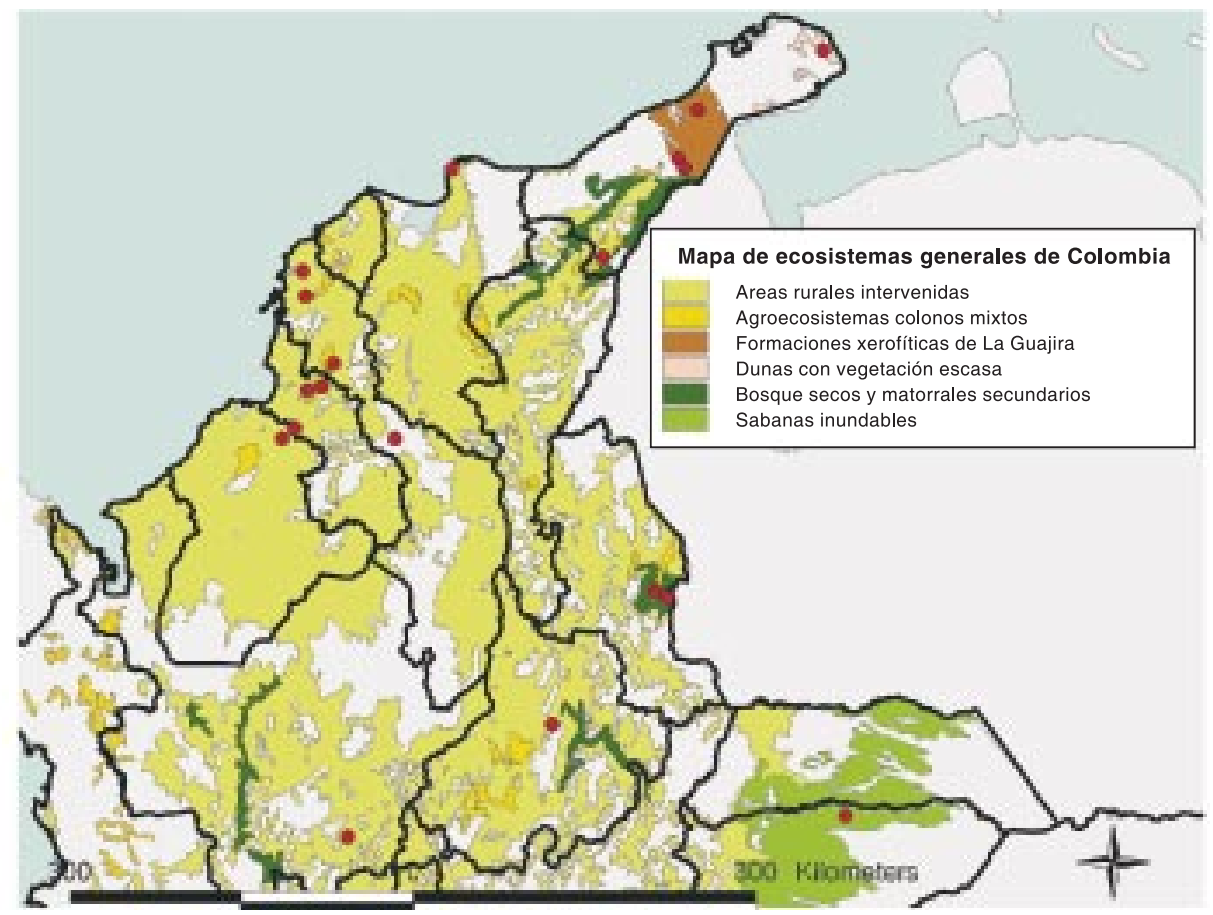

Figura 5. Distribución de Luitzomyia evansi de acuerdo con el mapa de ecosistemas generales de Colombia.

\section{Discusión}

Por primera vez, el Laboratorio de Entomología del Instituto Nacional de Salud presenta la distribución de los dos únicos vectores de leishmaniasis visceral en Colombia por medio de mapas digitales.

El control vectorial es una de las estrategias importantes en el manejo de enfermedades transmitidas por vectores; la elaboración de estos mapas se constituye en una herramienta de gran utilidad ya que permiten establecer las zonas en donde se encuentran los vectores $y$, por lo tanto, predecir a largo plazo posibles focos de aparición de la enfermedad.

L. longipalpis tiene una amplia distribución, principalmente, a lo largo del valle del río Magdalena mientras que $L$. evansi se localiza predominantemente en la Costa Atlántica. Las dos especies pueden encontrarse en las mismas localidades mostrando una distribución simpátrica. La mayor importancia epidemiológica de cada una de estas especies aparentemente está asociada con la abundancia de sus poblaciones, es decir,
L. longipalpis en el valle alto y medio del río Magdalena (38) y L. evansi en la Costa Atlántica. Sin embargo, es necesario profundizar en estudios ecológicos que permitan establecer los rangos climáticos determinantes de la misma.

Por medio de los mapas se estableció una asociación de la distribución de $L$. longipalpis y $L$. evansia localidades pertenecientes, especialmente, a la cobertura vegetal de bosque seco tropical; sin embargo, su distribución no está restringida a este tipo de bosque. Este hecho podría darse debido a que las dos especies en estudio tienen un comportamiento oportunista, de hábitos eclécticos que puede favorecer la colonización de nuevos ambientes y, por lo tanto, permitirles alcanzar una mayor distribución $(19,39)$. Por otro lado, también es importante tener en cuenta que la base de datos se elaboró a partir de registros de ejemplares recolectados en Colombia desde 1967; en algunos casos la asociación de las especies a las zonas de vida puede no ser tan precisa, dependiendo del grado de conservación de cada localidad en el momento del muestreo. Además, el alto grado de deforestación ha 
favorecido el aumento de algunas poblaciones de flebótomos en zonas cercanas a asentamientos rurales (21) y la domiciliación de los vectores puede darse de forma muy rápida $(18,21,23,40)$.

\section{Agradecimientos}

Proyecto: Biogeografía y genética de flebótomos vectores de enfermedades, Instituto Nacional de Salud-Yale University. Instituto Alexander von Humboldt, unidad de SIG.

\section{Conflicto de intereses}

Los autores declaran que no hubo ningún conflicto de intereses.

\section{Financiación}

Instituto Nacional de Salud y Yale University via U.S. National Institutes of Health, grant RO1 AI056254 para LEM.

\section{Referencias}

1. Desjeux P. Leishmaniasis: current situation and new perspectives. Comp Immunol Microbiol Infect Dis 2004;27:305-18.

2. Marty P, Rosenthal E. Treatment of visceral leishmaniasis: a review of current treatment practices. Expert Opin Pharmacother 2002;3:1101-8.

3. WHO. Control of leishmaniases. Report of a WHO Expert Committee. Technical Report Series, № 793. Geneva: World Health Organization; 1990.

4. Lane RP. Sandflies (Phlebotominae). En: Lane RP, Crosskey RW, editors. Medical insects and arachnids. Londres: Editorial Chapman \& Hall; 1996. p.78-119.

5. Duckworth D, Crandall R, Rathe R. Leishmania donovani. En: BUGS" Index-Organisms. Consultado: agosto 2003. Disponible en: http://medinfo.ufl.edu/ year2/mmid/bms5300/bugs/leidonov.html\#AA3.

6. Corredor A, Gallego JF, Tesh RB, Pelaez D, Díaz A, Montilla $\mathbf{M}$ et al. Didelphis marsupialis, an apparent wild reservoir of Leishmania donovani chagasi in Colombia, South America. Trans R Soc Trop Med Hyg 1989;83:195.

7. Corredor A, Gallego JF, Tesh RB, Morales A, De Carrasquilla CF, Young DG et al. Epidemiology of visceral leishmaniasis in Colombia. Am J Trop Med Hyg 1989;40:480-6.

8. Sherlock IA. Ecological interactions of visceral leishmaniasis in the state of Bahia, Brazil. Mem Inst Oswaldo Cruz 1996;91:671-83.

9. Travi BL, Tabares CJ, Cadena H, Ferro C, Osorio Y. Canine visceral leishmaniasis in Colombia: relationship between clinical and parasitologic status and infectivity for sand flies. Am J Trop Med Hyg 2001;64:119-24.

10. Young DG, Duncan MA. Guide to the identification and geographic distribution of Lutzomyia sand flies in Mexico, the West Indies, Central and South America (Diptera: Psychodidae). Mem Entomol Inst 1994;54:1881.

11. Ferro C, Morrison AC, Torres M, Pardo R, Wilson ML, Tesh RB. Age structure, blood-feeding behavior, and Leishmania chagasi infection in Lutzomyia longipalpis (Diptera: Psychodidae) at an endemic focus of visceral leishmaniasis in Colombia. J Med Entomol 1995;32:618-29.

12. Travi BL, Vélez ID, Brutus L, Segura I, Jaramillo C, Montoya J. Lutzomyia evansi, an alternate vector of Leishmania chagasi in a Colombian focus of visceral leishmaniasis. Trans R Soc Trop Med Hygiene 1990;84:676-7.

13. Dos Santos SO, Arias J, Ribeiro AA, de Paiva Hoffmann M, de Freitas RA, Malacco MA. Incrimination of Lutzomyia cruzi as a vector of American visceral leishmaniasis. Med Vet Entomol 1998;12:315-7.

14. Montoya-Lerma J, Ferro C. Flebótomos (Diptera: Psychodidae) de Colombia. En: Amat G, Andrade MG, Fernández $F$, editores. Insectos de Colombia. Volumen II. Colección Jorge Álvarez Lleras. № 13. Santa Fe de Bogotá: Academia Colombiana de Ciencias Exactas, Físicas y Naturales-Centro Editorial Javeriano; 1999. p. 211-45.

15. Young DG. A review of the bloodsucking psychodid flies of Colombia (Diptera: Phlebotominae and Sycoracinae). Technical Bulletin 806. Gainesville: Institute of Food and Agricultural Sciences, University of Florida; 1979. p.266.

16. Ibáñez-Bernal S, Rodríguez-Domínguez G, Gómez-Hernández $\mathrm{CH}$, Ricardez-Esquinca JR. First record of Lutzomyia evansi (Nuñez-Tovar 1924) in Mexico (Diptera: Psychodidae, Phlebotominae). Mem Inst Oswaldo Cruz 2003;99:127-9.

17. Travi BL, Adler GH, Lozano M, Cadena H, MontoyaLerma J. Impact of habitat degradation on phlebotominae (Diptera: Psychodidae) of tropical dry forests in Northern Colombia. J Med Entomol 2002;39:451-6.

18. Feliciangeli MD, Rodriguez N, De Guglielmo Z, Rodriguez A. The re-emergence of American visceral leishmaniasis in an old focus in Venezuela. II. Vectors and parasites. Parasite 1999;6:113-20.

19. Montoya-Lerma J, Cadena H, Oviedo M, Ready PD, Barazarte R, Travi BL et al. Comparative vectorial efficiency of Lutzomyia evansi and L. longipalpis for transmitting Leishmania chagasi. Acta Trop 2003; 85:19- 29. 
20. Montoya-Lerma J, Lane RP. Factors affecting host preference of Lutzomyia evansi (Diptera: Psychodidae), a vector of visceral leishmaniasis in Colombia. Bull Entomol Res 1996;86:43-50.

21. Tesh RB. Control of zoonotic visceral leishmaniasis: is it time to change strategies? Am J Trop Med Hyg 1995;52:287-92.

22. Bejarano EE, Uribe S, Rojas W, Vélez ID. Presence of Lutzomyia evansi, a vector of American visceral leishmaniasis, in an urban area of the Colombian Caribbean coast. Trans R Soc Trop Med Hyg 2001;95:27-8.

23. King RJ, Campbell-Lendrum DH, Davies CR. Predicting geographic variation in cutaneous leishmaniasis, Colombia. Emerg Inf Dis 2004;10:598607.

24. Morrison AC, Munstermann LE, Ferro C, Pardo R, Torres M. Ecological and genetic studies of Lutzomyia longipalpis in a central Colombia focus of visceral leishmaniasis. Bol Dir Malar San Amb1995;35 (Suppl.1):235-48.

25. Thompson RA, Wellington de Oliveira Lima J, Maguire JH, Braud DH, Scholl DT. Climatic and demographic determinants of American visceral leishmaniasis in northeastern Brazil using remote sensing technology for environmental categorization of rain and region influences on leishmaniasis. Am J Trop Med Hyg 2002;67:648-55.

26. Mullner RM, Chung K, Croke KG, Mensah EK. Geographic information systems in public health and medicine. J Med Syst 2004;28:215-21.

27. Osorno-Mesa E, Morales-Alarcon A, de Osorno F. Phlebotominae de Colombia (Diptera, Psychodidae) IVLutzomyia longipalpis (Lutz \& Neiva, 1912) en Colombia, S.A. Rev Acad Colomb Cienc 1969;13:379-82.

28. Young DG, Morales A, Kreutzer RD, Alexander JB, Corredor A, Tesh RB et al. Isolations of Leishmania braziliensis from criopreserved Colombian sand flies (Diptera: Psychodidae). J Med Entomol 1987;24:587-9.

29. Morales A, Ferro C, Isaza de Rodríguez C, Cura E. Encuesta sobre artrópodos de interés médico en La Guajira, Colombia, Suramérica. Biomédica 1987;7:8793.

30. López Y, Osorio L, Álvarez G, Rojas J, Jiménez F, Gómez $\mathbf{C}$ et al. Sandfly Lutzomyia longipalpis in a cutaneous leishmaniasis focus in central Colombia. Mem Inst Oswaldo Cruz 1996;91:415-9.
31. Vélez ID, Ghysais G, Marulanda J, Maya DA, Rivera I, Guerrero MA et al. Leishmaniasis tegumentaria americana: encuesta epidemiológica en una comunidad indígena. latreia 1988;1:29-33.

32. Bejarano EE, Sierra D, Vélez ID. Novedades en la distribución geográfica del grupo verrucarum (Diptera: Psychodidae) en Colombia. Biomédica 2003;23:34150.

33. Holdridge LR. Life zone ecology. San José de Costa Rica: Tropical Science Center; 1967. p.206.

34. Cavelier J. Selvas y bosques montanos. En: Chaves $\mathrm{S}$, Maria Elfi, Arango V, editores. Instituto de Investigación de Recursos Biológicos Alexander von Humboldt. Informe Nacional sobre el Estado de la Biodiversidad 1997, Colombia. Santafé de Bogotá: Instituto Humboldt, PNUMA, Ministerio del Medio Ambiente; 1998. p.38-55.

35. Espinal S. Zonas de vida o formaciones vegetales de Colombia, memoria explicativa sobre el mapa ecológico. Bogotá: Instituto Geográfico Agustín Codazzi; 1978.

36. Etter A. Mapa general de ecosistemas de Colombia. En: Chaves ME, Arango M, editores. Informe Nacional sobre el Estado de la Biodiversidad en Colombia. Tomo I. Diversidad Biológica. Instituto de Investigación de Recursos Biológicos Alexander von Humboldt, PNUMA, Bogotá: Ministerio del Medio Ambiente; 1998. p.1-150.

37. Campbell-Lendrum D, Dujardin JP, Martínez E, Feliciangeli MD, Pérez JE, Silans LN et al. Domestic and peridomestic transmission of American cutaneous leishmaniasis: changing epidemiological patterns present new control opportunities. Mem Inst Oswaldo Cruz 2000;96:159-62.

38. Morrison AC, Ferro C, Pardo R, Torres M, Devlin B, Wilson ML et al. Seasonal abundance of Lutzomyia longipalpis (Diptera: Psychodidae) at an endemic focus of visceral leishmaniasis in Colombia. J Med Entomol 1995;32:538-48

39. Morrison AC, Ferro C, Tesh RB. Host preferences of the sand fly Lutzomyia longipalpis at an endemic focus of American visceral leishmaniasis in Colombia. Am J Trop Med Hyg 1993;49;68-75.

40. Lainson R, Rangel EF. Ecología das Leishmanioses. En: Rangel EF, Lainson R, editores. Flebotomineos do Brasil. Rio de Janeiro: Editora Fiocruz; 2003. p.367. 